\title{
Antihyperglykämische Therapie bei Diabetes mellitus Typ 2 (Update 2019)
}

\author{
Martin Clodi · Heidemarie Abrahamian · Helmut Brath · Johanna Brix · Heinz Drexel · Peter Fasching · \\ Bernhard Föger · Claudia Francesconi · Elke Fröhlich-Reiterer · Jürgen Harreiter · Sabine E. Hofer · \\ Friedrich Hoppichler · Joakim Huber · Susanne Kaser · Alexandra Kautzky-Willer · Monika Lechleitner • \\ Bernhard Ludvik · Anton Luger · Julia K. Mader · Bernhard Paulweber · Thomas Pieber · Rudolf Prager • \\ Birgit Rami-Merhar - Michael Resl - Michaela Riedl - Michael Roden · Christoph H. Saely · Christian Schelkshorn • \\ Guntram Schernthaner · Harald Sourij - Lars Stechemesser · Harald Stingl · Hermann Toplak · \\ Thomas C. Wascher · Raimund Weitgasser · Yvonne Winhofer-Stöckl · Sandra Zlamal-Fortunat
}

Online publiziert: 12. April 2019

(C) Der/die Autor(en) 2019

M. Clodi $(\triangle) \cdot$ M. Resl

ICMR - Institute for Cardiovascular and Metabolic Research, Johannes Kepler Universität Linz, 4040 Linz, Österreich

Abteilung für Innere Medizin mit Diabetologie,

Gastroenterologie und Hepatologie, Rheumatologie und Intensivmedizin, Konventhospital der Barmherzigen Brüder Linz, Seilerstätte 2, 4021 Linz, Österreich martin.clodi@jku.at

\section{H. Abrahamian}

Internistisches Zentrum Baumgartner Höhe,

Otto-Wagner-Spital, Wien, Österreich

H. Brath

Diabetes Ambulanz, Gesundheitszentrum Wien-Süd, Wien, Österreich

J. Brix $\cdot$ B. Ludvik $\cdot$ G. Schernthaner

1. Medizinische Abteilung mit Diabetologie, Endokrinologie und Nephrologie, Krankenanstalt Rudolfstiftung, Wien,

Österreich

\section{H. Drexel}

Vorarlberg Institute for Vascular Investigation and

Treatment (VIVIT), Feldkirch, Österreich

Chair der ESC-Working Group „Cardiovascular

Pharmacotherapy“, Sophia Antipolis, Frankreich

Abteilung für Angiologie, Universitätsspital Bern, Bern,

Schweiz

Drexel University College of Medicine, Philadelphia, PA, USA

Private Universität im Fürstentum Liechtenstein,

Liechtenstein, Liechtenstein

P. Fasching

5. Medizinische Abteilung für Endokrinologie,

Rheumatologie und Akutgeriatrie, Wilhelminenspital der Stadt Wien, Wien, Österreich

\section{B. Föger}

Interne Abteilung, Landeskrankenhaus Bregenz, Bregenz,

Österreich

AKS Gesundheit, Bregenz, Österreich

\section{Francesconi}

Sonderkrankenanstalt Rehabilitationszentrum Alland, Alland, Österreich

E. Fröhlich-Reiterer

Universitätsklinik für Kinder- und Jugendheilkunde, Medizinische Universität Graz, Graz, Österreich

J. Harreiter · A. Luger · M. Riedl · Y. Winhofer-Stöckl Klinische Abteilung für Endokrinologie und Stoffwechsel, Universitätsklinik für Innere Medizin III, Medizinische Universität Wien, Wien, Österreich

\section{S. E. Hofer}

Department für Pädiatrie 1, Medizinische Universität Innsbruck, Innsbruck, Österreich

\section{F. Hoppichler}

Abteilung für Innere Medizin, Krankenhaus der Barmherzigen Brüder Salzburg, Salzburg, Österreich

J. Huber

Interne Abteilung mit Akutgeriatrie und Palliativmedizin, Franziskus Spital, Standort Landstraße, Wien, Österreich

\section{S. Kaser}

Department für Innere Medizin I, Medizinische Universität Innsbruck, Innsbruck, Österreich

Christian Doppler Labor für Insulinresistenz, Medizinische Universität Innsbruck, Innsbruck, Österreich

\section{A. Kautzky-Willer}

Gender Medicine Unit, Klinische Abteilung für Endokrinologie und Stoffwechsel, Universitätsklinik für Innere Medizin III, Medizinische Universität Wien, Wien, Österreich

\section{Lechleitner}

Interne Abteilung, Landeskrankenhaus Hochzirl - Natters, Hochzirl, Österreich

J. K. Mader · H. Sourij · H. Toplak

Klinische Abteilung für Endokrinologie und Diabetologie, Universitätsklinik für Innere Medizin, Medizinische Universität Graz, Graz, Österreich 
B. Paulweber · L. Stechemesser

Universitätsklinik für Innere Medizin I, mit

Gastroenterologie, Hepatologie, Nephrologie,

Stoffwechsel und Diabetologie, Paracelsus Medizinische

Privatuniversität, Salzburg, Österreich

\section{T. Pieber}

Universitätsklinik für Innere Medizin, Medizinische

Universität Graz, Graz, Österreich

R. Prager

3. Medizinische Abteilung mit Stoffwechselerkrankungen und Nephrologie, Krankenhaus Hietzing Wien, Wien, Österreich

\section{B. Rami-Merhar}

Universitätsklinik für Kinder- und Jugendheilkunde,

Medizinische Universität Wien, Wien, Österreich

\section{Roden}

Klinik für Endokrinologie und Diabetologie, Medizinische Fakultät, Heinrich-Heine-Universität, Düsseldorf, Deutschland

Deutsches Zentrum für Diabetesforschung, DZD e.V., München-Neuherberg, Deutschland

Institut für Klinische Diabetologie, Deutsches Diabetes-Zentrum (DDZ), Leibniz-Zentrum für Diabetesforschung, Düsseldorf, Deutschland

C. H. Saely

Abteilung für Innere Medizin I, Akademisches

Lehrkrankenhaus Feldkirch, Feldkirch, Österreich

\section{Schelkshorn}

1. Medizinische Abteilung, Landesklinikum Stockerau, Stockerau, Österreich

\section{H. Stingl}

Abteilung für Innere Medizin, Landesklinikum Melk, Melk, Österreich

T. C. Wascher

1. Medizinische Abteilung, Hanusch-Krankenhaus, Wien, Österreich

\section{R. Weitgasser}

Abteilung für Innere Medizin, Privatklinik

Wehrle-Diakonissen, Salzburg, Österreich

Universitätsklinik für Innere Medizin I, LKH SalzburgUniversitätsklinikum der Paracelsus Medizinischen Privatuniversität, Salzburg, Österreich

S. Zlamal-Fortunat

Abteilung für Innere Medizin und Gastroenterologie,

Klinikum Klagenfurt am Wörthersee, Klagenfurt, Österreich
Zusammenfassung Die Hyperglykämie ist wesentlich an der Entstehung der Spätkomplikationen bei an Diabetes mellitus Typ 2 erkrankten Patienten/ Patientinnen beteiligt. Während Lebensstilmaßnahmen die Eckpfeiler jeder Diabetestherapie bleiben, benötigen im Verlauf die meisten Patienten/Patientinnen mit Typ 2 Diabetes eine medikamentöse Therapie. Bei der Definition individueller Behandlungsziele stellen die Therapiesicherheit, die Effektivität sowie substanzspezifische, kardiovaskuläre Effekte der Therapie die wichtigsten Faktoren dar. In dieser Leitlinie haben wir die rezenten evidenzbasierten Daten für die klinische Praxis zusammengestellt.

Schlüsselwörter Diabetes mellitus Typ 2 - Therapie . Blutzuckersenkung

\section{Antihyperglycemic treatment guidelines for diabetes mellitus type 2 (Update 2019)}

Summary Hyperglycemia significantly contributes to complications in patients with diabetes mellitus. While lifestyle interventions remain cornerstones of disease prevention and treatment, most patients with type 2 diabetes will eventually require pharmacotherapy for glycemic control. The definition of individual targets regarding optimal therapeutic efficacy and safety as well as cardiovascular effects is of great importance. In this guideline we present the most current evidence-based best clinical practice data for healthcare professionals.

Keywords Diabetes mellitus Type 2 - Therapy · Glucose lowering

\section{Einleitung}

Die Hyperglykämie der an Diabetes mellitus Typ 2 erkrankten Patienten trägt entscheidend zur Pathogenese mikrovaskulärer Komplikationen bei und ist Cofaktor bei der Entwicklung makrovaskulärer Erkrankungen.

Das primäre Ziel einer antihyperglykämischen Therapie ist daher das Vermeiden von akuten und chronischen Komplikationen der Hyperglykämie auf Basis der Verhinderung von Veränderungen im Zellstoffwechsel.

Des Weiteren stellen Symptomfreiheit, der Erhalt der Lebensqualität, sowie Komaprophylaxe wesentliche Therapieziele dar.

Der Zusammenhang zwischen Hyperglykämie und Mikroangiopathie ist im Vergleich zur Makroangiopathie stärker ausgeprägt und linear. Damit ist eine Prävention durch verbesserte Blutzuckereinstellung im Bereich der Mikroangiopathie leichter zu erreichen. 


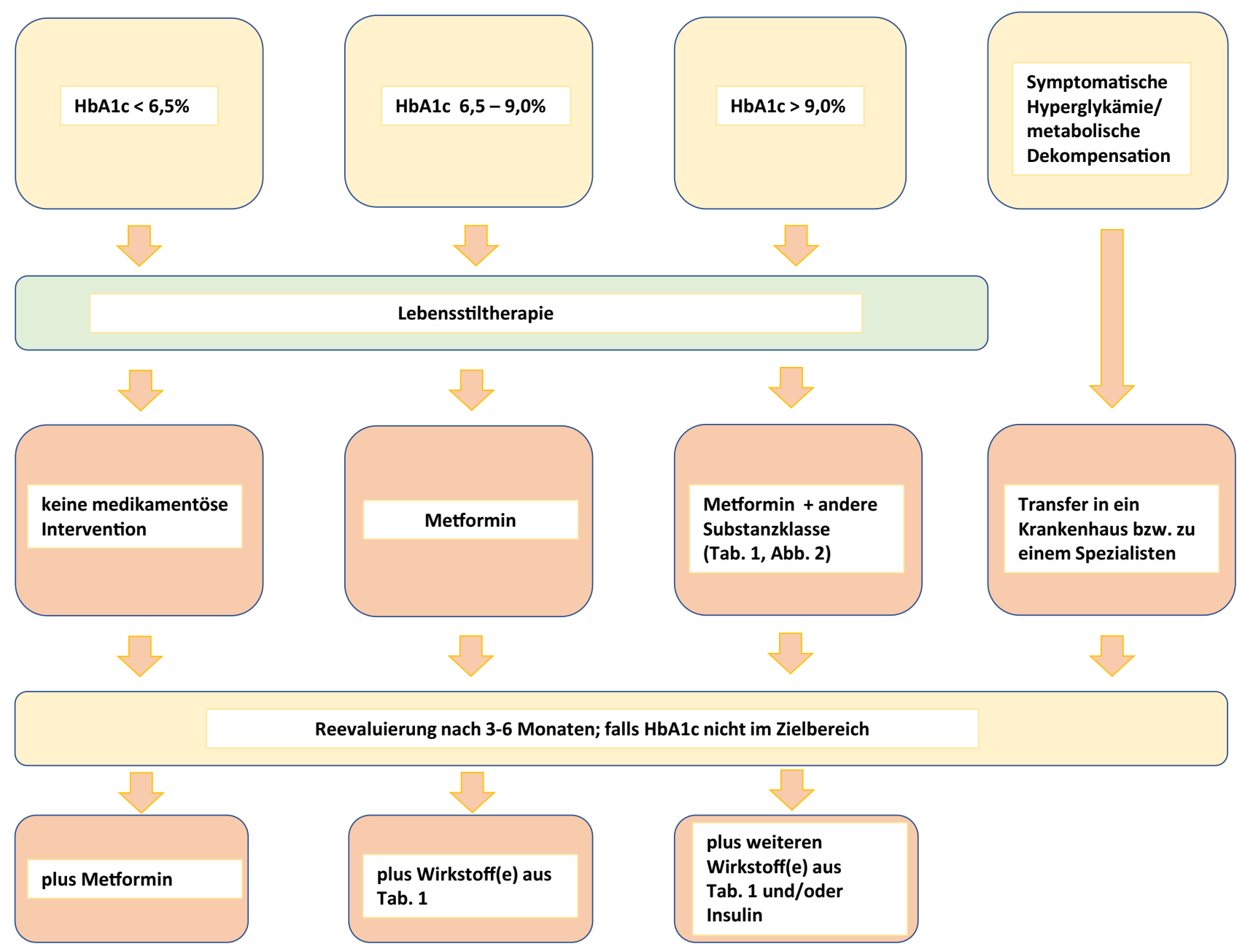

Abb. 1 Therapie nach rezenter Diagnose Diabetes mellitus Typ 2

\section{Therapieziele}

$\mathrm{Zu}$ den allgemeinen Zielen der Therapie zählen:

- Vermeiden von Akutkomplikationen,

- Vermeiden von Folgekomplikationen,

- Symptomfreiheit sowie Erhalt bzw. Wiederherstellung der Lebensqualität.

Bei Prädiabetes wird basierend auf der aktuell verfügbaren Datenlage die Umsetzung lebensstilmodifizierender Maßnahmen mit dem Ziel einer Gewichtsreduktion, Verbesserung der Ernährungsqualität und Steigerung der körperlichen Fitness empfohlen. Eine medikamentöse Therapie mit Metformin kann insbesondere bei Menschen unter 60 Jahren, bei einem BMI $\geq 35 \mathrm{~kg} / \mathrm{m}^{2}$, bei positiver Familienanamnese, bei hohen Triglyzeriden und niedrigem HDL-Cholesterin und Frauen mit vorangegangenem Gestationsdiabetes in Erwägung gezogen werden.

Für die antihyperglykämische Therapie gelten unten angeführte Zielwerte. Als Mittel der ersten Wahl soll Metformin eingesetzt werden. Bei einer Kontraindikation oder einer Unverträglichkeit gegenüber Metformin muss je nach individuellen Erfordernissen des
Patienten ein anderes der verfügbaren Präparate angewandt werden.

Sollte bei einer Monotherapie mit einem Medikament der Zielwert nicht erreicht werden, muss eine Therapiemodifikation durchgeführt werden. Möglichkeiten hierfür sind in den Abb. 1 und 2 zum Teil in Analogie zu den aktuell gültigen Leitlinien der Amerikanischen und Europäischen Diabetesgesellschaft dargestellt [1]. Weiterhin existieren nur wenige Daten zur Auswahl der Medikamente bei Triplekombinationen.

Große, randomisiert kontrollierte Studien der letzten Jahre konnten substanzspezifische kardiovaskuläre und renale Effekte unterschiedlichen Ausmaßes bei Gliflozinen (Empagliflozin > Canagliflozin > Dapagliflozin) wie auch bei GLP-1 Analoga (Liraglutid $>$ Semaglutid) dokumentieren.

Wird unter Metformin der individuell festgelegte HbAlc-Zielwert nicht erreicht, so wird bei der erforderlichen Therapieeskalation die Berücksichtigung kardiovaskulärer Komorbiditäten empfohlen. Letztlich sollte bei bereits manifester kardiovaskulärer Erkrankung ein Präparat mit substanzspezifischen, 


\section{leitlinien für die praxis}

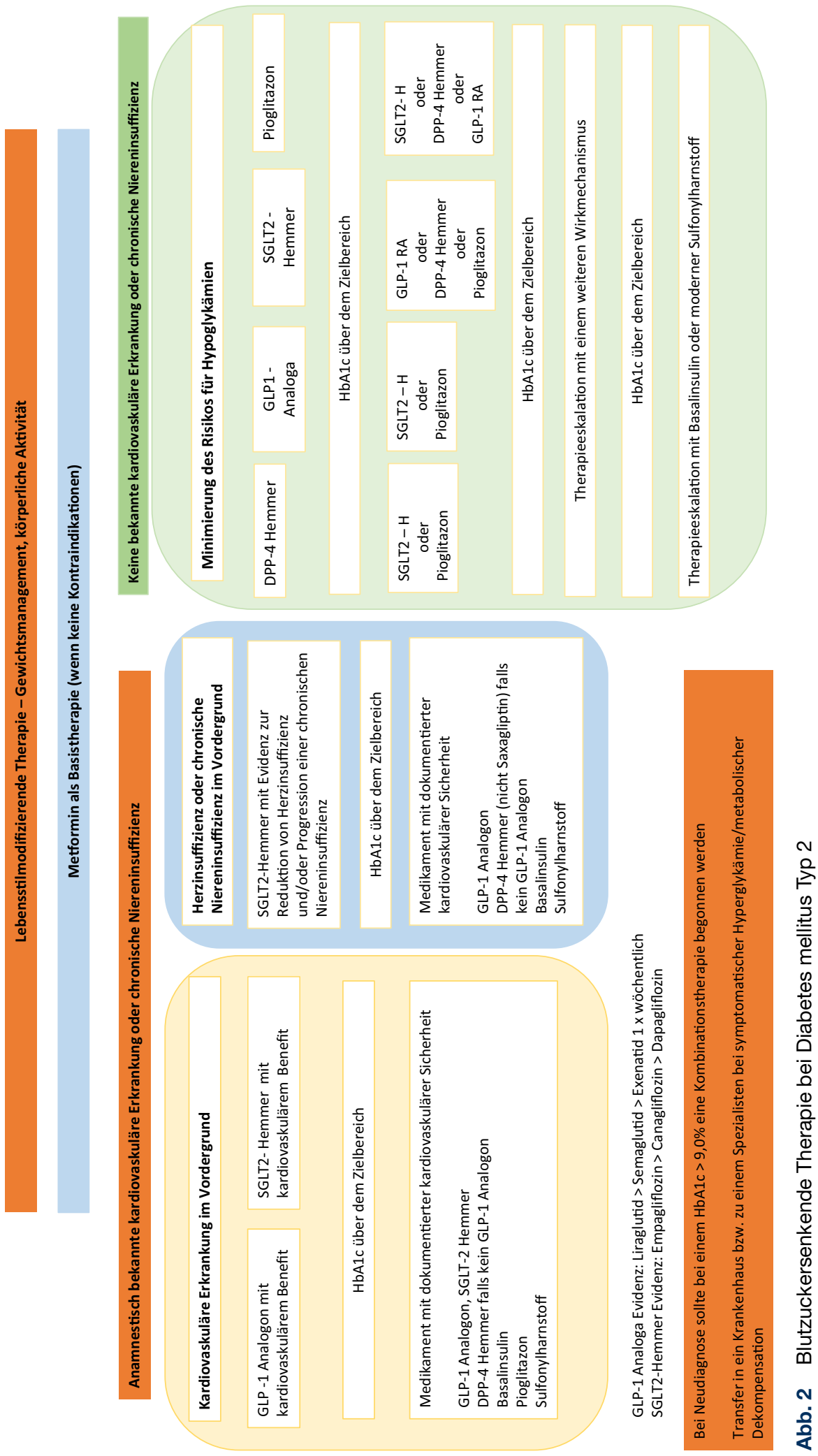


positiven kardiovaskulären Effekten als Erweiterung der Metformintherapie ausgewählt werden.

Das HbAlc stellt die primäre Richtgröße der Stoffwechselkontrolle dar. Postprandiale Glukose und Nüchternglukose stellen weitere Richtgrößen dar.

\section{FACT-Box}

- Basis jeder Diabetestherapie ist eine lebenslange Lebensstilmodifikation (Gewichtsreduktion/Bewegung).

- Bei kurzer Diabetesdauer und langer Lebenserwartung wird ein HbAlc-Zielwert < 6,5\% empfohlen, sofern das ohne relevante Nebenwirkungen der Therapie erreicht werden kann.

- Ein HbAlc-Ziel kleiner 7,0\% ist für einen ausreichenden mikro- und makrovaskulären Schutz notwendig.

- Metformin nimmt nach wie vor eine zentrale Rolle in der Behandlung ein.

- Derzeit gibt es für Empagliflozin, Canagliflozin, Dapagliflozin, Liraglutid, Semaglutid und Pioglitazon (sekundärer Endpunkt) publizierte, positive, substanzspezifische kardiovaskuläre Daten aus placebokontrollierten, randomisierten prospektiven Studien (RCT).

Generell ist für die meisten Patienten ein $\mathrm{HbAlc}<7,0 \%$ für einen ausreichenden mikrovaskulären, makrovaskulären und zellulären Schutz notwendig.

Bei Patienten mit kurzer Diabetesdauer, langer Lebenserwartung und keiner relevanten kardiovaskulären Komorbidität ist ein HbAlc-Wert unter 6,5\% sinnvoll.

Bei Patienten mit mehreren, schweren Hypoglykämien und/oder eingeschränkter Lebenserwartung sowie multiplen Spätkomplikationen kann ein HbAlcZielwert bis zu 8,0\% als ausreichend erachtet werden.

Neben dem HbAlc stellen die Nüchtern- und die postprandiale Glukose sekundäre Richtgrößen dar. Dementsprechend sollte die Nüchternglukose unter $130 \mathrm{mg} / \mathrm{dl}$ (ideal $<110 \mathrm{mg} / \mathrm{dl}$ ) liegen und die postprandiale Glukose ( $2 \mathrm{~h}$ nach einer Mahlzeit) maximal $180 \mathrm{mg} / \mathrm{dl}$ betragen.

\section{Antidiabetika im Überblick}

\section{Metformin}

Metformin (Tab. 1) wirkt primär durch eine Hemmung der Glukoneogenese mit Senkung der (Nüchtern)Glukoseproduktion, nachfolgend tritt eine Verbesserung der hepatischen und peripheren Insulinsensitivität ein. In der Monotherapie wird durch Metformin eine HbAlc-Reduktion von ca. 1,5\% bei einer Senkung des Nüchternblutzuckers um $30-40 \mathrm{mg} / \mathrm{dl}$ erreicht. Die Metformintherapie wird mit zweimal $500 \mathrm{mg}$ pro Tag begonnen und sollte langsam (1-2 wöchentlich) bis zu $2000 \mathrm{mg}$ gesteigert werden. Ge- nerell ist auch bei übergewichtigen, geriatrischen Patienten eine initiale Therapie mit Metformin $\mathrm{zu}$ empfehlen. Der appetithemmende und damit gewichtsreduzierende Effekt (bis $\mathrm{zu}-2 \mathrm{~kg}$ über mehrere Jahre) von Metformin kann aber gerade beim geriatrischen Patienten aufgrund der Gefahr einer Malnutrition unerwünscht sein (siehe Geriatrie-Leitlinie). Gastrointestinale Nebenwirkungen werden bei dieser schrittweisen Steigerung der Tagesdosis reduziert. Als Kontraindikationen für die Metformintherapie gelten eine schwere Einschränkung der Nierenfunktion, dekompensierte Lebererkrankungen, akute Pankreatitis, Alkoholismus, Malnutrition, eine dekompensierte Herzinsuffizienz und/oder andere hypoxische Situationen. Metformin darf bei Patienten/Patientinnen mit eGFR-Werten zwischen $30-45 \mathrm{ml} / \mathrm{min} / 1,73 \mathrm{~m}^{2}$ bei Fehlen von anderen Risikofaktoren für Laktatazidosen in einer Dosierung von $1000 \mathrm{mg}$, täglich aufgeteilt auf zwei Dosen, angewandt werden. Die glomeruläre Filtrationsrate sollte mit Hilfe einer entsprechenden Formel (zum Beispiel CKD-EPI oder MDRD) evaluiert zumindest alle 3-6 Monate kontrolliert werden. Falls die eGFR unter $30 \mathrm{ml} / \mathrm{min} / 1,73 \mathrm{~m}^{2}$ abfällt, muss Metformin abgesetzt werden. Bei interkurrierenden schweren Erkrankungen (schwere Infektionen) sowie auch bei Diarrhoe und Exsikkose und der Applikation von jodhaltigen Kontrastmitteln soll Metformin ebenso vorübergehend pausiert werden. Da es unter einer Langzeittherapie mit Metformin zu einem Vitamin B12-Mangel kommen kann, wird empfohlen, die Vitamin B12-Spiegel in regelmäßigen Abständen zu kontrollieren, vor allem wenn eine Anämie oder eine periphere Neuropathie vorliegt.

\section{SGLT2-Inhibitoren}

Der Natrium-Glucose-Cotransporter (SGLT2) ist verantwortlich für den größten Teil der Glukose-Resorption im proximalen Tubulus der Niere. Die SGLT2Inhibitoren bewirken daher eine kontrollierte Glukosurie und damit eine Reduktion der Hyperglykämie. Die Wirkung der SGLT-2-Hemmer ist unabhängig von Insulin. Die aktuell verfügbaren Substanzen sind Dapagliflozin, Empagliflozin, Canagliflozin und Ertugliflozin. Prinzipiell können SGLT-2-Hemmer in jeder Kombination eingesetzt werden. Neben der Blutzuckersenkung (das HbAlc sinkt um 0,5-1\%) kommt es zu einer Senkung des Blutdruckes (2-4/1-2 mm Hg) und $\mathrm{zu}$ einer Gewichtsabnahme $(-2-3 \mathrm{~kg})$, wobei das LDL-Cholesterin gering ansteigt (etwa 5\%). Aufgrund der glukosurischen Wirkung dieser Medikamentenklasse ergibt sich auch ein diuretischer Effekt. Unter Therapie mit Gliflozinen wurde vereinzelt das Auftreten von euglykämischen Ketoazidosen berichtet [2]. Laut einer FDA-Warnung vom Dezember 2015 gibt es dafür potenzielle Risikofaktoren: Infektionen, „Low-Carbohydrate Diet“, Reduktion/Absetzen einer laufenden Insulintherapie, Absetzen von oralen Insulinsekretagoga, Diabetes mellitus Typ 1 und Alkohol- 
Tab. 1 Bewertung oraler Antidiabetika und von Insulin

\begin{tabular}{|c|c|c|c|c|}
\hline Klasse & HbA1c (in \%) & Hypoglykämie & Vorteile & Nachteile \\
\hline Metformin & $1-2$ & Nein & $\begin{array}{l}\text { Gewichtsneutralität, Reduktion makrovaskulärer Ereignis- } \\
\text { se }\end{array}$ & KI und GI Nebenwirkungen \\
\hline \multicolumn{5}{|c|}{ Hinzufügen eines Wirkstoffes, der für den einzelnen Patienten auf Basis der unten angeführten Vor- und Nachteile am besten geeignet ist } \\
\hline SGLT-2-Hemmer & $0,5-1$ & Nein & $\begin{array}{l}\text { Empagliflozin und Canagliflozin reduzieren kardiovaskulä- } \\
\text { re Ereignisse, Reduktion der Mortalität durch Empagliflo- } \\
\text { zin; Dapagliflozin reduziert den kombinierten Endpunkt } \\
\text { (Tod und Hospitalisierung wegen Herzinsuffizienz); Ge- } \\
\text { wichtsreduktion }\end{array}$ & $\begin{array}{l}\text { Genitale Infekte, sehr selten Auslöser } \\
\text { normoglykämischer Ketoazidosen, Hin- } \\
\text { weise auf erhöhtes Amputationsrisiko } \\
\text { (für Canagliflozin) }\end{array}$ \\
\hline $\begin{array}{l}\text { GLP-1-Rezeptor- } \\
\text { Agonisten }\end{array}$ & $1-2$ & Nein & $\begin{array}{l}\text { Gewichtsreduktion, Reduktion kardiovaskulärer Ereignis- } \\
\text { se unter Liraglutid und Semaglutid; Mortalitätsreduktion } \\
\text { unter Liraglutid }\end{array}$ & Nausea, subkutane Injektion \\
\hline Pioglitazon & $1-2$ & Nein & Reduktion kardiovaskulärer Ereignisse & $\begin{array}{l}\text { Gewichtszunahme, periphere Ödeme, } \\
\text { Herzinsuffizienz, Frakturen bei Frauen }\end{array}$ \\
\hline DPP-4-Hemmer & $0,5-1$ & Nein & Gewichtsneutral & $\begin{array}{l}\text { Gefahr der Hospitalisierung wegen } \\
\text { Herzinsuffizienz (Saxagliptin) }\end{array}$ \\
\hline Sulfonylharnstoffe & $1-2$ & $\mathrm{Ja}$ & Rasche Blutzuckersenkung & $\begin{array}{l}\text { Mögliche Gewichtszunahme, Hypogly- } \\
\text { kämien }\end{array}$ \\
\hline Glinide & $1-2$ & $\mathrm{Ja}$ & Verbesserte postprandiale BZ-Kontrolle & $\begin{array}{l}\text { Dreimal tägliche Dosierung, mögliche } \\
\text { Gewichtszunahme }\end{array}$ \\
\hline $\begin{array}{l}\text { Alpha-Glucosidase- } \\
\text { Inhibitoren }\end{array}$ & $0,5-1,0$ & Nein & Verbesserte postprandiale BZ-Kontrolle, gewichtsneutral & $\begin{array}{l}\text { Gl Nebenwirkungen, mäßige Wirksam- } \\
\text { keit }\end{array}$ \\
\hline Insulin & 2 & $\mathrm{Ja}$ & Keine Dosisobergrenze, viele Arten, flexible Regelungen & Gewichtszunahme, Hypoglykämie \\
\hline
\end{tabular}

missbrauch. Sollte sich eine Ketoazidose bestätigen, müssen die SGLT-2-Hemmer sofort abgesetzt und eine stationäre Therapie eingeleitet werden. Generell sollen Gliflozine bei Operationen und/oder schweren Erkrankungen pausiert werden.

Ertugliflozin ist durch die Behörden bereits zugelassen worden, die entsprechenden, kardiovaskulären Endpunktstudien werden derzeit gerade durchgeführt.

\section{GLP-1-Rezeptor Agonisten}

Glucagon-like Peptide-1 (GLP-1) Rezeptor Agonisten (Exenatid, Liraglutid, Semaglutid, Lixisenatid, Dulaglutid) führen zu einer Glukose-abhängigen Steigerung der pankreatischen Insulinsekretion, Hemmung der Glucagonfreisetzung und der Magenentleerung sowie Auslösung eines Sättigungsgefühls durch Stimulation des Sättigungszentrums im Gehirn. Sie müssen subkutan in Intervallen von einmal täglich bis einmal wöchentlich (Exenatid LAR, Dulaglutid, Semaglutid) je nach Substanz verabreicht werden. Neben effektiver Blutzuckerreduktion sind die fehlende Hypoglykämieneigung und eine, in Studien beobachtete, Gewichtsreduktion festzuhalten. Gastrointestinale Nebenwirkungen (Übelkeit, Erbrechen) treten häufiger auf als unter Placebo.

\section{Pioglitazon}

Pioglitazon erhöht die Insulinsensitivität als Ligand der nukleären Hormonrezeptorfamilie PPAR- $\Upsilon$, über die Regulation der Expression verschiedener insulinempfindlicher Gene. Im Fettgewebe erfolgt eine verstärkte Differenzierung von Präadipozyten zu Adi- pozyten und damit eine Beeinflussung der metabolischen und endokrinen Aktivität. Die Insulinsensitivität in Leber, Skelettmuskel und im Fettgewebe nimmt zu. In Abhängigkeit vom Ausgangs-HbAlc-Wert und der Dosierung reduzieren Glitazone den HbAlc-Wert um etwa 1,5\%. Zu den Nebenwirkungen der Glitazontherapie zählen Gewichtszunahme und verstärkte Ödemneigung auf Basis von Flüssigkeitsretention. Kontraindikationen für die Glitazontherapie sind Herzinsuffizienz (wegen Flüssigkeitsretention durch erhöhte Natrium-Rückresorption) und Leberfunktionsstörungen. Pioglitazon selbst hat keine direkten negativen, kardialen Effekte. Bei postmenopausalen Frauen wurde eine Steigerung traumatischer Knochenbrüche beobachtet.

\section{Gliptine}

Dipeptidyl-Peptidase-IV-Inhibitoren (Sitagliptin, Vildagliptin, Saxagliptin, Linagliptin, Alogliptin) als Abbauhemmer des körpereigenen GLP-1 führen zu einer Glukose-abhängigen Steigerung der pankreatischen Insulinsekretion und Hemmung der Glucagonfreisetzung. Diese Substanzen zeigen keine Hypoglykämieneigung und sind gewichtsneutral. Aufgrund der relativ geringen Wirkung in der Monotherapie werden sie in Kombination mit Metformin (primär), anderen OADs oder aber in Tripelkombination eingesetzt. In Kombination mit Metformin wird eine substanzeigene HbAlc-Senkung von ca. $0,8 \%$ beobachtet.

In den RCTs fand sich eine numerisch erhöhte Inzidenz für Pankreatitis im Promillebereich, diese erreichte jedoch in den Einzelstudien keine statistische Signifikanz. 


\section{Sulfonylharnstoffe}

Sulfonylharnstoffe (Gliclazid, Glimepirid, Gliquidon) stimulieren die pankreatische Insulinsekretion und resultieren in einer mittleren zu erwartenden HbAlcReduktion um 1,5\%. $\mathrm{Zu}$ den klinisch relevanten Nebenwirkungen zählt das erhöhte Hypoglykämierisiko. Gliclazid hat ein niedrigeres Hypoglykämierisiko im Vergleich zu den meisten anderen Sulfonylharnstoffen. In den letzten Jahren wurden Sulfonylharnstoffe in Zusammenhang mit einem erhöhten kardiovaskulären Risiko gebracht, welches in Metaanalysen für die angeführten Sulfonylharnstoffe nicht bestätigt werden konnte.

\section{Glinide}

Glinide (Repaglinid) führen zu einer gegenüber Sulfonylharnstoffderivaten kürzer dauernden prandialen Insulinsekretion mit einer größeren Flexibilität hinsichtlich der Nahrungsaufnahme. Analog zu den Sulfonylharnstoffen besteht jedoch auch ein Hypoglykämierisiko. Die HbAlc-Reduktion in der Monotherapie beträgt ca. 1,0\%. Diese Präparate sind unmittelbar präprandial einzunehmen. Positive, kardiovaskuläre Endpunktstudien liegen für die Glinide nicht vor.

\section{Alpha-Glucosidase-Inhibitoren}

Diese Substanzklasse, deren wichtigster Vertreter Acarbose ist, bewirkt über eine Hemmung der intestinalen Kohlenhydratverdauung eine Reduktion vor allem der postprandialen Blutzuckerwerte um $50-60 \mathrm{mg} / \mathrm{dl}$ und des HbAlc-Werts um 0,7\%. Acarbose kann sowohl als Monotherapie als auch als Therapieergänzung eingesetzt werden. Nebenwirkungen dieser Therapie (Blähungen und Bauchschmerzen) können durch eine schrittweise Dosissteigerung und individuelle Anpassung verringert werden.

\section{Insuline}

Generell stellt die Basalinsulintherapie eine einfache und gleichzeitig auch sichere Möglichkeit für den Einstieg in eine Insulintherapie dar. Kann unter dieser Therapie das individuell festgelegte Therapieziel nicht erreicht werden, so sollte je nach Wünschen und Bedürfnissen des Patienten eine Intensivierung der Therapie mithilfe eines zusätzlich verabreichten, prandialen Insulins oder mittels Mischinsulin erfolgen (siehe Insulintherapie-Leitlinie.

\section{Evidenzlage}

\section{Blutzuckersenkung}

Der epidemiologische Zusammenhang zwischen dem Ausmaß der Hyperglykämie und dem Auftreten mikro- und makrovaskulärer sowie zellulärer Komplikationen ist gesichert.

Die zentrale Evidenz der UKPDS ist, dass eine intensivierte Therapie mit Insulin oder Sulfonylharnstoffen einer konventionellen Therapie mit primär Diät im Hinblick auf Komplikationen überlegen ist, wobei eine Verbesserung des HbAlc um 0,9\% erreicht wurde [3].

Ein spezifischer Substanzvorteil stellte sich nur für Metformin als Therapie der 1. Wahl bei übergewichtigen Patienten dar. In dieser Gruppe wurden Myokardinfarkte sowie Diabetes-assoziierte Mortalität und Gesamtmortalität signifikant gesenkt [4]. Die Follow-up-Untersuchung der UKPDS-Population legt nahe, dass durch intensivierte Therapie langfristig die Gesamtmortalität gesenkt werden kann [5]. Ebenso legt diese Untersuchung die Existenz eines „metabolischen Gedächtnisses“ bei frisch manifestierten Patienten mit Typ 2 Diabetes nahe.

UKPDS [5], ADVANCE [6], ACCORD und VADT [7, 8] legen zusammenfassend nahe, dass eine gute Blutzuckerkontrolle durch intensivierte Therapiestrategien, möglichst unmittelbar nach Diagnosestellung, erreicht und ohne schwere Hypoglykämien und exzessive Gewichtszunahme aufrechterhalten werden sollte. Langzeitbeobachtungen der Studien haben gezeigt, dass eine kardiovaskuläre Risikoreduktion von ca. $20 \%$ erreicht werden kann [9]. In diesen Studien hatten die Patientengruppen mit den tiefsten HbAlc-Werten auch die niedrigste Mortalitätsrate pro Jahr.

\section{Substanzspezifische, kardiovaskuläre Effekte}

\section{SGLT-2-Hemmer}

Die EMPA-REG Outcome-Studie [10] zeigte eine signifikante Reduktion des primären Endpunktes (kardiovaskulärer Tod, nicht-tödlicher Myokardinfarkt oder nicht-tödlicher Insult) durch Empagliflozin verglichen mit Placebo (HR 0,86 CI 0,74-0,99, $p=0,04$ ). Kardiovaskulärer Tod (HR 0,62 CI 0,49-0,77, $p<0,001$ ) und Gesamtmortalität (HR 0,68; CI 0,57-0,82; $p<0,001$ ) wurden bei den in der Studie eingeschlossenen Patienten mit kardiovaskulären Vorerkrankungen (KHK $75 \%$, Herzinfarkt $43 \%$, Schlaganfall $26 \%$, PAVK 23\%, Herzinsuffizienz 10\%) unter Empagliflozin signifikant im Vergleich zu Placebo gesenkt. Auch die Hospitalisierungsrate für Herzinsuffizienz sank unter Empagliflozin um ein Drittel (HR 0,65; CI 0,50-0,85; $p=0,002)$, das Auftreten von nicht-tödlichem Herzinfarkt, instabiler Angina pectoris und nicht-tödlichem Schlaganfall wurden hingegen nicht signifikant beeinflusst. Besonders bemerkenswert ist, dass die Effekte trotz Vorbehandlung (75\% Metformin, 48\% Insulin, 75\% Statine, 80\% ACE/ARB, 90\% Plättchen-Inhibitoren; $25 \%$ CABG) nachweisbar waren und zeitlich bereits sehr frühzeitig (nach 3-6 Monaten) auftraten. In weiterführenden Analysen konnte letztlich auch eine Reduktion renaler Endpunkte (Progression zu Ma- 
kroalbuminurie, Verdoppelung des Serumkreatinins in Verbindung mit einer eGFR $\leq 45 \mathrm{ml} / \mathrm{min} / 1,73 \mathrm{~m}^{2}$, Neubeginn einer Nierenersatztherapie oder renaler Tod) (HR 0,61; CI 0,53-0,70; $p<0,001$ ) dokumentiert werden [11]. Beinahe analog zu Empagliflozin zeigte auch Canagliflozin eine signifikante Reduktion des präspezifizierten Endpunktes (HR 0,86; CI 0,75-0,97, $p=0,02)$. Im Vergleich zu EMPA-REG Outcome betrug die Prävalenz der kardiovaskulären Erkrankung in CANVAS 65,6\% [12].

Die substanzspezifische, kardiovaskuläre Sicherheit von Dapagliflozin wurde in der DECLARE-TIMI 58 Studie an 17.160 Patienten untersucht, wobei 10.186 Patienten am Beginn der Studie keine kardiovaskuläre Erkrankung aufwiesen. Im Rahmen der primären Ergebnisanalyse konnte die kardiovaskuläre Sicherheit von Dapagliflozin bestätigt werden. Darüber hinaus wurde eine signifikante Reduktion des kombinierten Endpunktes aus kardiovaskulären Todesfällen und Hospitalisationen aufgrund von Herzinsuffizienz registriert (HR 0,83; CI 0,73-0,95; $p=0,005)$. Dieser Effekt war hauptsächlich durch eine Reduktion der Hospitalisierung durch Herzinsuffizienz getrieben. Die Reduktion von Hospitalisierung wegen Herzinsuffizienz konnte auch in der Gruppe mit multiplen Risikofaktoren (Dyslipidämie, Hypertonie, Rauchen) ohne vorhergehende kardiovaskuläre Erkrankung nachgewiesen werden [13].

\section{GLP1-Analoga}

Auch für Liraglutid liegen positive Daten aus einer kardiovaskulären Endpunktstudie vor. In der LEADERStudie bewirkte Liraglutid eine signifikante Reduktion kardiovaskulärer Todesfälle und weiterführend eine Reduktion des präspezifizierten Endpunktes (HR 0,87; CI 0,78-0,97; $p=0,01$ für superiority). Des Weiteren konnte ein präspezifizierter, kombinierter renaler Endpunkt ebenso signifikant reduziert werden. Am Beginn der Studie hatten $81 \%$ der Patienten eine bekannte kardiovaskuläre Erkrankung [14].

Für Semaglutid bestätigt die SUSTAIN-6 Studie ebenfalls positive kardiovaskuläre Effekte. Der primäre Endpunkt trat in der Semaglutid-Gruppe signifikant geringer auf (HR 0,74; CI 0,58-0,95 $p<0,001$ für Non-Inferiority), wobei nicht tödliche Schlaganfälle in der Semaglutidgruppe signifikant reduziert wurden (HR 0,61; CI 0,38-0,99; $p=0,04$ ) Entgegen den positiven kardiovaskulären Effekten kam es unter Semaglutid zu gehäuften Manifestationen der diabetischen Retinopathie (Glaskörperblutung, Erblindung, intravitreale Injektionen oder Photokoagulation) (HR 1,76; CI 1,11-2,78, $p=0,02$ ) [15].

\section{PPAR-Gamma-Agonisten}

Die Datenlage für Pioglitazon ist hinsichtlich einer möglichen kardiovaskulären Prävention ebenso positiv. Für Pioglitazon existiert mit PROACTIVE eine positive Endpunktstudie [16], die hinsichtlich des sekundären Endpunktes schwerer kardiovaskulärer Ereignisse insgesamt und besonders für die Subgruppen der Patienten mit vorangegangenem Myokardinfarkt [17] oder Schlaganfall [18] deutliche Vorteile zeigt. Dieser sekundäre Endpunkt war gleich wie der primäre Endpunkt in den rezenten Cardiovascular Outcome Trials. Der primäre Endpunkt der PROACTIVE-Studie, der auch weniger harte kardiovaskuläre Ereignisse wie Amputationen einschloss, wurde nicht signifikant reduziert. Eine Metaanalyse für Pioglitazon unterstützt die möglichen kardiovaskulär präventiven Eigenschaften [19]. Die TOSCA.ITStudie verglich Pioglitazon mit Sulfonylharnstoffen (Glimepirid, Gliclazid) zusätzlich zu einer bereits etablierten Metformintherapie hinsichtlich eines kombinierten, kardiovaskulären Endpunktes (Gesamtmortalität, nicht tödlicher Myokardinfarkt, nicht tödlicher Insult, dringliche Revaskularisation eines Koronargefäßes). Zusammenfassend konnte kein signifikanter Unterschied zwischen den unterschiedlichen Therapiestrategien (HR 0,96; 95\% CI 0,74-1,26, $p=0,79$ ) dokumentiert werden [20]. In der IRIS-Studie, welche bei Patienten mit rezentem Insult und Insulinresistenz allerdings ohne manifestem Diabetes mellitus Typ 2 durchgeführt wurde, bewirkte eine Therapie mit Pioglitazon eine signifikante Reduktion des Auftretens von erneuten ischämischen Insulten und Myokardinfarkt (HR 0,76; CI 0,62-0,93; $p=0,007$ ) [21].

\section{Gliptine}

Rezente Endpunktstudien belegen die kardiovaskuläre Sicherheit von Sita-, Saxa- Lina- und Alogliptin. Es konnte aber kein kardiovaskulärer Zusatznutzen nachgewiesen werden. Im Rahmen der SAVOR-TIMI53-Studie wurde unter einer Therapie mit Saxagliptin eine signifikant höhere Rate von Hospitalisierungen aufgrund von Herzinsuffizienz beobachtet. Dieses Resultat bestätigte sich jedoch in den Studien für Sitaund Alogliptin nicht [22-24].

\section{Acarbose}

Im Rahmen der ACE-Studie konnte kein direkter, substanzspezifischer, kardiovaskulärer Zusatznutzen nachgewiesen werden [25].

\section{Basalinsulinanaloga}

Unter einer Basalinsulintherapie mit Glargin konnte in der ORIGIN-Studie bei rezent an Diabetes mellitus Typ 2 erkrankten Patienten keine signifikante Reduktion kardiovaskulärer Endpunkte gezeigt werden. Unter Glargin kam es aber auch zu keiner Zunahme kardiovaskulärer Ereignisse. Insulin Glargin kann somit als kardiovaskulär sicher angesehen werden. Interessanterweise waren im Vergleich zur Kontrollgruppe die Hypoglykämierate und die Gewichtszunahme 

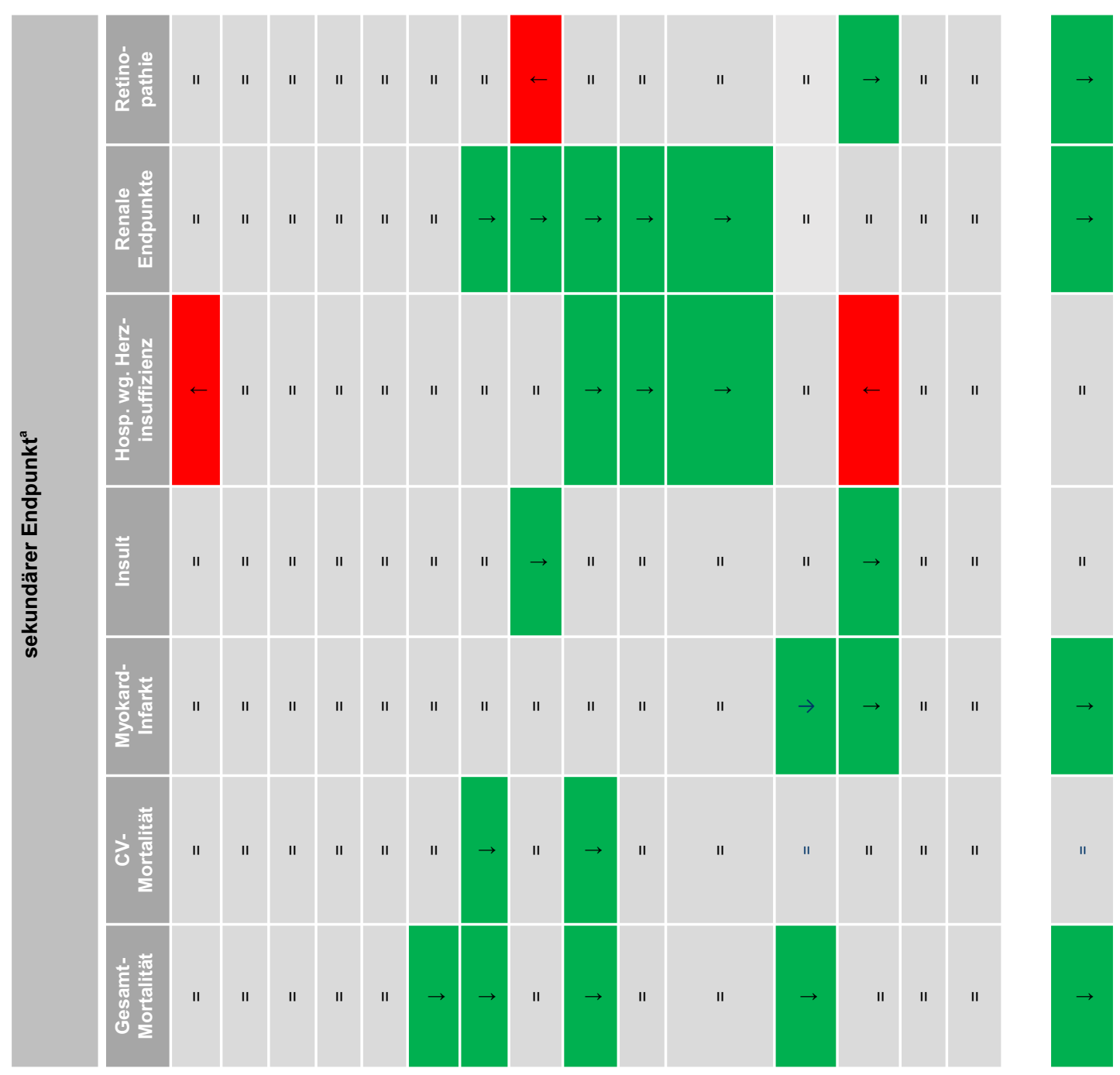

ब่

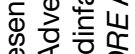

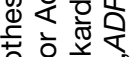
范

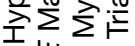
*

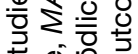

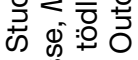
듄 는 는

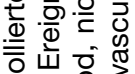

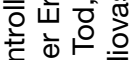

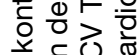
응 过䒓这

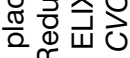

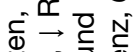

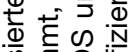
雪 을 需 $\cong \frac{\overline{0}}{\mathrm{~T}}$ 乐嵌 ब

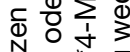
西 준

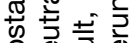
क人 क बे के क्षे

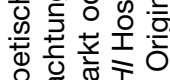

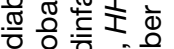

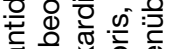

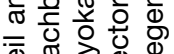

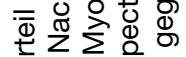

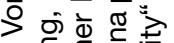

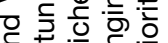

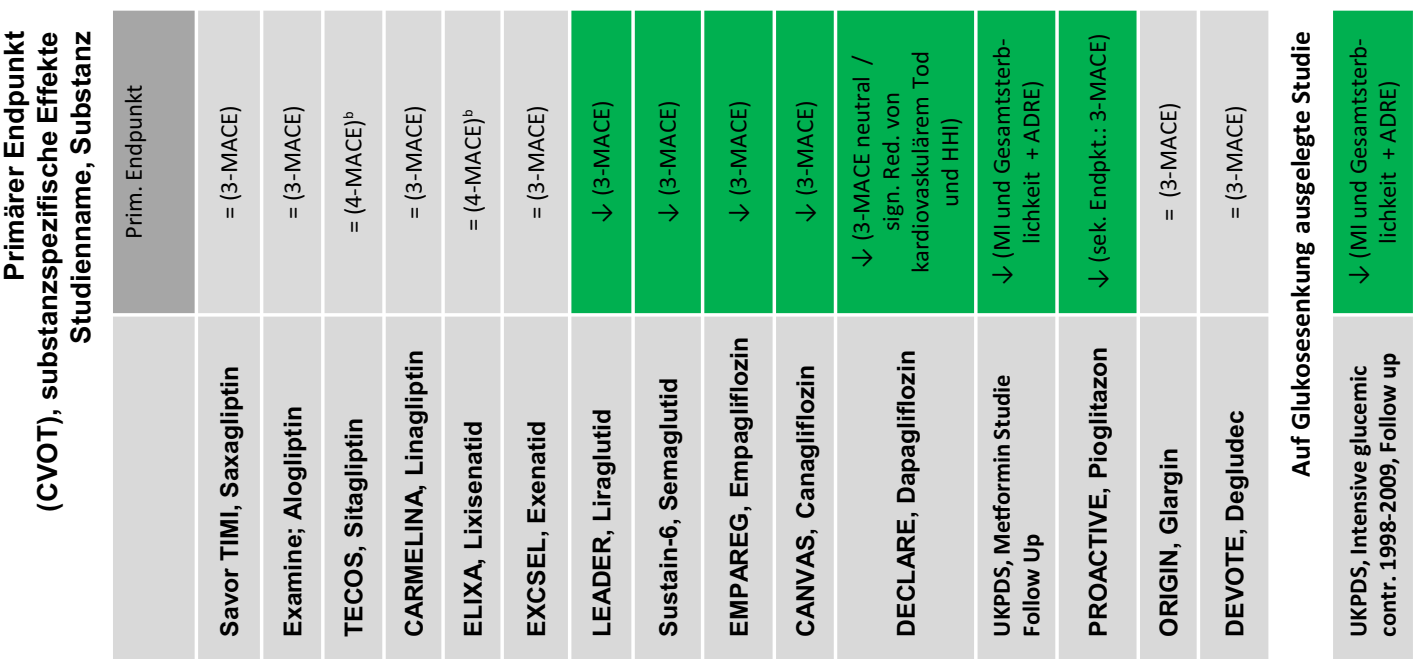
苾过这

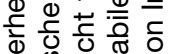
o 证 के

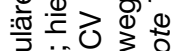

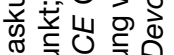

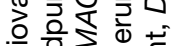
응 응 $\sum_{i} \frac{0}{0}+\vec{c}$ 훈 गे

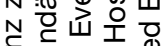
웡

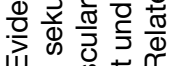
山. $\infty$ क वे की

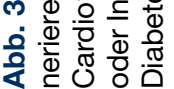


zwar signifikant erhöht, aber nur gering ausgeprägt [26]. Im Vergleich zu Glargin konnte auch für Insulin Degludec die kardiovaskuläre Sicherheit gezeigt werden [27].

Studiendaten zu kardiovaskulärer Sicherheit und Vorteile antidiabetischer Substanzen aus randomisierten, placebokontrollierten Studien sind in Abb. 3 angeführt.

Funding Open access funding provided by Johannes Kepler University Linz.

Interessenkonflikt Abrahamian Heidemarie hat Vortragshonorare und Honorare für Beratungstätigkeit von folgenden Firmen erhalten: Eli Lilly, Astra Zeneca, Boehringer Ingelheim, Amgen, Sanofi, Novo Nordisk, Servier, MSD. Helmut Brath erhielt Vortragshonorare und Honorare von folgenden Firmen: AstraZeneca, Boehringer Ingelheim, Amgen, Sanofi, Novo Nordisk, Servier, Eli Lilly, MSD, Takeda, Gebro, Genericon, Abbott. Johanna Brix hat Vortragshonorare und Honorare für Beratungstätigkeit von folgenden Firmen erhalten: AstraZeneca, Bayer, Boehringer Ingelheim, Eli Lilly, MSD, Novartis, Novo Nordisk. Martin Clodi hat Vortragshonorare und Honorare für Beratungstätigkeit von folgenden Firmen erhalten: Eli Lilly, AstraZeneca, Boehringer Ingelheim, Amgen, Sanofi, Novo Nordisk, Servier. Heinz Drexel hat in den letzten 36 Monaten von folgenden Unternehmen, die teils auch fördernde Mitglieder der ÖDG sind, Forschungsunterstützungen und/oder Honorare erhalten: Amgen, Bayer Austria, Boehringer Ingelheim, Meda Pharma, Merck Sharp \& Dohme, Novo Nordisk, sanofi-aventis, Servier. Peter Fasching hat Vortragshonorare und Honorare für Beratungstätigkeit von folgenden Firmen erhalten: AstraZeneca, Boehringer Ingelheim, MSD, Novartis, Takeda, Servier, Eli Lilly, Novo Nordisk, Sanofi. Bernhard Föger hat in den letzten 36 Monaten von folgenden Unternehmen, die teils auch fördernde Mitglieder der ÖDG sind, Forschungsunterstützungen und/oder Honorare erhalten: Alexion, Amgen, AOP Orphan, AstraZeneca; Boehringer Ingelheim, Eli Lilly, Janssen-Cilag, MEDA, Merck Sharp \& Dohme, Mylan, Novartis, Novo Nordisk, Roche, sanofi-aventis. Claudia Francesconi hat Vortragshonorare und Honorar für Beratungstätigkeit von folgenden Firmen erhalten: Boehringer Ingelheim, AstraZeneca, Novo Nordisk, Lilly, Sanofi, MSD, Takeda, Dexcom. Elke Fröhlich-Reiterer hat in den letzten 36 Monaten von folgenden Firmen Honorare/ Forschungsgelder erhalten: Medtronic, Novo Nordisk, Eli Lilly, Roche. Jürgen Harreiter erhielt Forschungsförderungen oder Honorare von folgenden Firmen: AstraZeneca, Novo Nordisk, Takeda. Sabine Hofer hat in den letzten 36 Monaten von folgenden Firmen Honorare/Forschungsgelder erhalten: Eli Lilly, sanofi. Friedrich Hoppichler hat in Bezug auf diesen Artikel von folgenden Unternehmen, die teils auch fördernde Mitglieder der ÖDG sind, Forschungsunterstützungen und/oder Honorare erhalten: AstraZeneca, Boehringer Ingelheim, Janssen, Eli Lilly, Merck Sharp \& Dohme, Novartis, Novo Nordisk, Sanofi, Servier, Takeda. Joakim Huber hat von folgenden Unternehmen, die auch fördernde Mitglieder der ÖDG sind, Fortbildungsunterstützung und/oder Honorare für Vorträge und Beratung erhalten: Eli Lilly, Novartis, Novo Nordisk, Boehringer Ingelheim, AstraZeneca, sanofi-aventis, Merck Sharp \& Dohme, Roche, Daiichi-Sankyo, Takeda. Zusätzlich gibt J. Huber an, dass für ihn persönlich kein Interessenkonflikt vorliegt. Susanne Kaser hat von folgenden Unternehmen, die auch fördernde Mitglieder der ÖDG sind, Forschungsunterstützungen und/oder Honorare erhalten: AstraZeneca, Boehringer Ingelheim, Eli Lilly, Merck Sharp \& Dohme, Novartis, Novo Nordisk, Roche, sanofi-aventis,
Servier, Takeda. Alexandra Kautzky-Willer hat von folgenden Unternehmen, die auch fördernde Mitglieder der ÖDG sind, Forschungsunterstützungen und/oder Honorare erhalten: AstraZeneca, Amgen, Boehringer Ingelheim, Eli Lilly, Merck Sharp \& Dohme, Novartis, Novo Nordisk, Roche, sanofi-aventis, Servier, Takeda. Monika Lechleitner hat von folgenden Unternehmen, die auch fördernde Mitglieder der ÖDG sind, Honorare für Vorträge und Advisory Boards erhalten: Boehringer, Novo, Sanofi. Bernhard Ludvik hat von folgenden Unternehmen, die auch fördernde Mitglieder der ÖDG sind, Forschungsunterstützungen und/oder Honorare erhalten: Abbott Austria, Aengus, Amgen, AstraZeneca, Bayer, Boehringer Ingelheim, Eli Lilly, Medtronic, Merck Sharp \& Dohme, Novartis, Novo Nordisk, Roche, sanofi-aventis, Servier, Takeda. Anton Luger hat von folgenden Unternehmen, die auch fördernde Mitglieder der ÖDG sind, Forschungsunterstützungen und/oder Honorare erhalten: Boehringer Ingelheim, Eli Lilly, MSD, Novartis, Novo Nordisk, sanofi-aventis, Takeda. Julia Mader erhielt Honorare von: NovoNordisk, sanofiaventis, Nintamed, Eli Lilly, Takeda, Merck Sharp \& Dohme, AstraZeneca, Boehringer Ingelheim, Medtronic Minimed, Roche Diabetes Care. Forschungsgelder: A. Menarini Diagnostics, BBraun, Metronom Health, NovoNordisk, Roche Diabetes Care, sanofi-aventis. Bernhard Paulweber hat von folgenden Unternehmen, die auch fördernde Mitglieder der ÖDG sind, Forschungsunterstützungen und/oder Honorare erhalten: Abbott, Amgen, AstraZeneca, Bayer, Boehringer Ingelheim, Bristol-Myers Squibb, Eli Lilly, Genzyme, GlaxoSmith-Kline Pharma, Medtronic, Merck, Sharp \& Dohme, Novartis, Novo Nordisk, sanofi-aventis, Takeda, Unilever. Thomas Pieber: Direktor des Institutes für Biomedizin und Gesundheitswissenschaften (HEALTH) von JOANNEUM RESEARCH. Wissenschaftlicher Vorstand von CBmed (Zentrum für Biomarkerforschung). Vortragshonorare von der Firma Novo Nordisk, Mitglied von Advisory Boards von den Firmen Arecor, Novo Nordisk, Sanofi, AstraZeneca, Adocia. Rudolf Prager hat in den letzten 36 Monaten von folgenden Firmen Honorare/Forschungsgelder erhalten: Amgen, AstraZeneca, Boehringer Ingelheim, Bristol-Myers Squibb, Eli Lilly, Janssen, Medtronic, Merck-Sharp \& Dohme, Novartis, Novo Nordisk, sanofi-aventis, Takeda. Birgit RamiMerhar hat in den letzten 36 Monaten von folgenden Firmen Honorare/Forschungsgelder erhalten: Eli Lilly, Medtronic, Novo Nordisk, Menarini, Roche. Michael Resl hat Vortragshonorare und Honorare für Beratungstätigkeit von folgenden Firmen erhalten Boehringer Ingelheim, sanofi-aventis, Novo Nordisk. Michaela Riedl hat in den letzten 36 Monaten von folgenden Firmen Honorare/Forschungsgelder erhalten: AstraZeneca, Boehringer Ingelheim, Eli Lilly, Medtronic, Novo Nordisk, sanofi-aventis. Michael Roden hat von folgenden Unternehmen Forschungsunterstützungen und/ oder Honorare erhalten: AstraZeneca, Boehringer Ingelheim, Eli Lilly, Genentech, Merck, Novartis, Novo Nordisk, Nutricia/Danone, Poxel, sanofi-aventis. Christoph H. Saely hat von folgenden Unternehmen, die auch fördernde Mitglieder der ÖDG sind, Forschungsunterstützungen und/oder Honorare erhalten: Amgen, AstraZeneca, Boehringer Ingelheim, Eli Lilly, Merck, MSD, Mylan, Novartis, Novo Nordisk, sanofi-aventis, Servier, Takeda. Christian Schelkshorn hat von folgenden Unternehmen, die auch fördernde Mitglieder der ÖDG sind, Forschungsunterstützungen und/oder Honorare erhalten: Abbott, AstraZeneca, Boehringer Ingelheim, Bristol Myers Squibb, Daiichi Sankyo, Eli Lilly, Germania, GlaxoSmithKline Pharma, Medtronic, Merck, Sharp \& Dohme, Novartis, Novo Nordisk, sanofi-aventis, Servier, Ypsomed. Guntram Schernthaner hat Lectures gehalten für: AstraZeneca, Boehringer Ingelheim, Eli Lilly, Mundipharma, 
Novo Nordisk, Servier, Takeda. Sourij Harald hat von folgenden Unternehmen, die auch fördernde Mitglieder der ÖDG sind, Forschungsunterstützungen und/oder Honorare erhalten: Amgen, AstraZeneca, Eli Lilly, Merck Sharp \& Dohme, Novo Nordisk, sanofi-aventis, Takeda. Lars Stechemesser hat von folgenden Unternehmen, die teils auch fördernde Mitglieder der ÖDG sind, Forschungsunterstützungen und/oder Honorare erhalten: Abbott, AstraZeneca, Boehringer Ingelheim, EliLilly, Medtronic, MSD, Novartis, Novo Nordisk, Roche, sanofi-aventis. Harald Stingl legt offen, dass er von folgenden Unternehmen, die teils auch fördernde Mitglieder der ÖDG sind, Forschungsunterstützungen, Honorare für Vorträge und/oder Beratertätigkeit, bzw. Fortbildungsunterstützung im Rahmen der (dienst)rechtlichen Rahmenbedingungen (Einladung zu Kongressen) erhalten hat: Amgen, AstraZeneca, Boehringer Ingelheim, Eli Lilly, Gebro, Menarini, MSD, Novo Nordisk, Novartis, sanofi-aventis, Servier, Takeda. Zusätzlich gibt H. Stingl an, dass für ihn persönlich kein Interessenkonflikt vorliegt. Hermann Toplak hat von folgenden Unternehmen, die teils auch fördernde Mitglieder der ÖDG sind, Forschungsunterstützungen und/ oder Honorare erhalten: Abbott, AstraZeneca, Boehringer Ingelheim, Gebro, Eli Lilly, Milan, MSD, Novartis, Novo Nordisk, Pfizer, Roche, Sanofi, Servier, Takeda. Thomas Wascher hat von folgenden Unternehmen, die teils auch fördernde Mitglieder der ÖDG sind, Forschungsunterstützungen und/ oder Honorare erhalten: Abbott, Amgen, Boehringer Ingelheim, Eli Lilly, Merck Sharp \& Dohme, Mylan, Novo Nordisk, sanofi-aventis, Takeda. Raimund Weitgasser hat von folgenden Unternehmen, die teils auch fördernde Mitglieder der ÖDG sind, Forschungsunterstützungen und/oder Honorare erhalten: Abbott, Allergan, Astra Zeneca, Boehringer-Ingelheim, Dexcom, Eli Lilly, MSD, Novo Nordisk, Roche, Sanofi, Servier, Takeda. Yvonne Winhofer-Stöckl hat von folgenden Firmen, die auch teils auch fördernde Mitglieder der ÖDG sind, Honorare für Vorträge und Beratung erhalten: Amgen, AstraZeneca, Eli Lilly, Novartis, Sanofi. Sandra Zlamal-Fortunat hat von folgenden Unternehmen, die teils auch fördernde Mitglieder der ÖDG sind, Forschungsunterstützungen und/ oder Honorare erhalten: AstraZeneca, Boehringer Ingelheim, Bristol-Myers, Eli Lilly, Medtronic, Novartis, Novo Nordisk, Roche, sanofi-aventis, Servier, Takeda, Hipp.

Open Access Dieser Artikel wird unter der Creative Commons Namensnennung 4.0 International Lizenz (http:// creativecommons.org/licenses/by/4.0/deed.de) veröffentlicht, welche die Nutzung, Vervielfältigung, Bearbeitung, Verbreitung und Wiedergabe in jeglichem Medium und Format erlaubt, sofern Sie den/die ursprünglichen Autor(en) und die Quelle ordnungsgemäß nennen, einen Link zur Creative Commons Lizenz beifügen und angeben, ob Änderungen vorgenommen wurden.

\section{Literatur}

1. Davies MJ, et al. Management of hyperglycaemia in type 2 diabetes, 2018. A consensus report by the American Diabetes Association (ADA) and the European Association for the Study of Diabetes (EASD). Diabetologia. 2018;61(12):2461-98.

2. Peters AL, et al. Euglycemic Diabetic Ketoacidosis: A Potential Complication of Treatment With SodiumGlucose Cotransporter 2 Inhibition. Diabetes Care. 2015;38(9):1687-93.

3. Intensive blood-glucose control with sulphonylureas or insulin compared with conventional treatment and risk of complications in patients with type 2 diabetes (UKPDS 33).
UK Prospective Diabetes Study (UKPDS) Group. Lancet, 1998.352(9131):p. 837-53.

4. Effect of intensive blood-glucose control with metformin on complications in overweight patients with type 2 diabetes (UKPDS 34). UK Prospective Diabetes Study (UKPDS) Group. Lancet, 1998.352(9131):p. 854-65.

5. Holman RR, et al. 10-year follow-up of intensive glucose control in type 2 diabetes. N Engl J Med. 2008;359(15):157789.

6. Group AC, et al. Intensive blood glucose control and vascular outcomes in patients with type 2 diabetes. N Engl J Med. 2008;358(24):2560-72.

7. Action to Control Cardiovascular Risk in Diabetes Study, G., etal., Effects of intensive glucoselowering in type 2 diabetes. NEngl J Med, 2008.358(24):p. 2545-59.

8. Duckworth W, et al. Glucose control and vascular complications in veterans with type 2 diabetes. N Engl J Med. 2009;360(2):129-39.

9. Ferrannini E, DeFronzo RA. Impact of glucose-lowering drugs on cardiovascular disease in type 2 diabetes. Eur Heart J.2015;36(34):2288-96.

10. Zinman B, et al. Empagliflozin, Cardiovascular Outcomes, and Mortality in Type 2 Diabetes. N Engl J Med. 2015;373(22):2117-28.

11. Wanner C, et al. Empagliflozin and Progression of Kidney Disease in Type 2 Diabetes. N Engl J Med. 2016;375(4):323-34.

12. Neal B, Perkovic V, Matthews DR. Canagliflozin and Cardiovascular and Renal Events in Type 2 Diabetes. N Engl J Med. 2017;377(21):2099.

13. Wiviott SD, et al. Dapagliflozin and Cardiovascular Outcomesin Type2Diabetes. NEnglJMed.2019;380(4):347-357.

14. Marso SP, et al. Liraglutide and Cardiovascular Outcomes in Type 2 Diabetes. NEngl J Med. 2016;375(4):311-22.

15. Marso SP, Holst AG, Vilsboll T. Semaglutide and Cardiovascular Outcomes in Patients with Type 2 Diabetes. N Engl J Med.2017;376(9):891-2.

16. DormandyJA, etal. Secondary prevention of macrovascular events in patients with type 2 diabetes in the PROactive Study (PROspective pioglitAzone Clinical Trial In macroVascular Events): a randomised controlled trial. Lancet. 2005;366(9493):1279-89.

17. Erdmann E, et al. The effect of pioglitazone on recurrent myocardial infarction in 2,445 patients with type 2 diabetes and previous myocardial infarction: results from the PROactive (PROactive 05) Study. J Am Coll Cardiol. 2007;49(17):1772-80.

18. Wilcox R, et al. Effects of pioglitazone in patients with type 2 diabetes with or without previous stroke: results from PROactive (PROspective pioglitAzone Clinical Trial In macroVascular Events 04). Stroke. 2007;38(3):865-73.

19. Lincoff AM, et al. Pioglitazone and risk of cardiovascular events in patients with type 2 diabetes mellitus: a metaanalysis of randomized trials. JAMA. 2007;298(10):1180-8.

20. Vaccaro $O$, et al. Effects on the incidence of cardiovascular events of the addition of pioglitazone versus sulfonylureas in patients with type 2 diabetes inadequately controlled with metformin (TOSCA.IT): a randomised, multicentre trial. Lancet Diabetes Endocrinol. 2017;5(11):887-97.

21. KernanWN, etal. Pioglitazoneafter Ischemic Stroke or Transient Ischemic Attack. NEngl J Med. 2016;374(14):1321-31.

22. Green JB, et al. Effect of Sitagliptin on Cardiovascular Outcomes in Type 2 Diabetes. NEngl J Med.2015;373(3):232-42.

23. Scirica BM, et al. Saxagliptin and cardiovascular outcomes in patients with type 2 diabetes mellitus. N Engl J Med. 2013;369(14):1317-26. 


\section{leitlinien für die praxis}

24. White WB, et al. Alogliptin after acute coronary syndrome in patients with type 2 diabetes. N Engl J Med. 2013;369(14):1327-35.

25. Holman RR, et al. Effects of acarbose on cardiovascular and diabetes outcomes in patients with coronary heart disease and impaired glucose tolerance (ACE): a randomised, double-blind, placebo-controlled trial. Lancet Diabetes Endocrinol.2017;5(11):877-86.
26. Investigators OT, et al. Basal insulin and cardiovascular and other outcomes in dysglycemia. N Engl J Med. 2012;367(4):319-28.

27. Marso SP, Buse JB. Safety of Degludec versus Glargine in Type2 Diabetes. NEngl J Med.2017;377(20):1995-6.

Hinweis des Verlags Der Verlag bleibt in Hinblick auf geografische Zuordnungen und Gebietsbezeichnungen in veröffentlichten Karten und Institutsadressen neutral. 